\title{
Philosophiques
}

\section{Le rôle de l'action dans la constitution du monde chez Husserl et Heidegger}

\section{Dagfinn Føllesdal}

Volume 20, numéro 2, automne 1993

Perspectives sur la phénoménologie et l'intentionnalité

URI : https://id.erudit.org/iderudit/027226ar

DOI : https://doi.org/10.7202/027226ar

Aller au sommaire du numéro

Éditeur(s)

Société de philosophie du Québec

ISSN

0316-2923 (imprimé)

1492-1391 (numérique)

Découvrir la revue

Citer cet article

Føllesdal, D. (1993). Le rôle de l'action dans la constitution du monde chez Husserl et Heidegger. Philosophiques, 20(2), 267-284.

https://doi.org/10.7202/027226ar d'utilisation que vous pouvez consulter en ligne.

https://apropos.erudit.org/fr/usagers/politique-dutilisation/ 

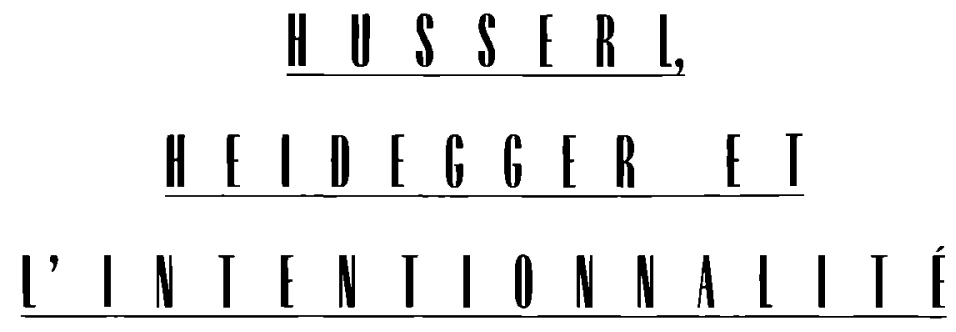

\section{LE RÔLE DE L'ACTION DANS LA CONSIITUTION DU MONDE CHEZ HUSSERL ET HEIDEGGER'}

\section{par \\ Dagfinn Follesdal}

Cet article a pour but de préciser les positions prises par Husserl et Heidegger sur le rôle que joue l'activité humaine dans la constitution du monde. Alors que l'idée fondamentale de la phénoménologie husserlienne veut que nous constituions le monde par notre conscience, la principale contribution de Heidegger à la philosophie, il me semble, est d'avoir mis l'accent sur le fait que l'ensemble de l'activité humaine, toutes les manières par lesquelles nous sommes en relation avec le monde, les autres et nous-mêmes, contribuent à constituer le monde.

Ceci représente selon moi un progrès significatif par rapport à la pensée de Husserl. Toutefois, nous retrouvons, surtout dans les manuscrits inédits de Husserl, certains passages indiquant

Je remercie Elling Schwabe-Hansen pour l'aide qu'il m’apporta dans la recherche de passages dans les manuscrits de Husserl permettant d'éclairer sa position, ainsi que le Conseil de Recherche Norvégien pour la Science et les Humanités pour son support généreux à cette recherche. Je suis également reconnaissant envers $\mathrm{Hu}^{-}$ bert Dreyfus. Harrison Hall et Samuel Todes pour leur commentaire sur une précédente version de cet article présenté lors du symposium de l'Association Aménicaine de Philosophie, division ouest, qui eut lieu à Cincinnati du 27 au 29 avilil et où ces derniers étaient invités à titre de commentateurs. J'ai également été aidé par les commentaires de Robert Nozick et de John Perry. 
qu'il considérait aussi que nos actions et l'activité pratique contribuent à notre constitution du monde. Nous examinerons ces passages ultérieurement. Je commencerai tout d'abord par exposer, de façon générale et en m’appuyant uniquement sur leurs écrits publiés, les positions de Husserl et de Heidegger sur la constitution, ainsi que leurs divergences de vues sur ce point.

\section{La position de Hussers Surr la constitution : l'intentionnalité}

Pour Husserl, « constitution » est un autre terme pour désigner l'intentionnalité de la conscience. Comme nous le verrons, Husserl soutenait, tout comme son maître Brentano, que la conscience est caractérisée par un certain type de directionalité : il semble toujours y avoir quelqu'objet vers lequel la conscience est dirigée, et dont nous avons conscience. Lorsque nous pensons, il semble toujours y avoir quelque chose à quoi nous pensons, quand nous percevons, une chose que nous percevons, etc., et de même pour tout autre type d'acte ou d'activité mentale. Brentano tenta de clarifier cette notion de directionalité en mettant l'accent sur l'objet, qu'il nomme « objet intentionnel », mais il fit face à de sérieuses difficultés dans les cas où de tels objets n'existent pas, les cas d'hallucinations par exemple, ou lorsque quelqu'un pense à quelque chose comme Pégase. On pourrait tenter de surmonter ces difficultés en maintenant que les objets de notre conscience ne sont pas réels, mais qu'ils sont plutôt contenus d'une certaine façon dans notre propre conscience, peu importe ce que cela peut signifier. Cette solution entraîne cependant certaines difficultés dans le cas de plusieurs autres actes, comme par exemple dans le cas d'actes de perception normale. Il semble que de ce point de vue, lorsque nous voyons un arbre, ce n'est pas l'arbre réel nous faisant face que nous voyons, mais quelque chose d'autre que nous aurions également vu si nous avions été victimes d'une hallucination. Ainsi, la position suivant laquelle tout acte est dirigé vers un objet conduit à un dilemme.

Husserl résout ce dilemme en proposant une analyse de la conscience d'après laquelle il n'est pas nécessaire qu'il y ait un objet vers lequel l'acte soit dirigé, mais où l'accent est plutôt mis sur ce en quoi consiste la directionalité, sur la recherche des caractéristiques de la conscience faisant en sorte que celle-ci se comporte toujours comme si elle était conscience de quelque 
chose. Ainsi, dans le cas de la perception, Husserl s'intéresse aux caractéristiques de la conscience faisant qu'un acte de perception soit comme s'il était perception d'un objet de tel et tel type, situé de telle et telle manière par rapport aux autres objets et à celui qui le perçoit. Husserl s'intéresse également aux caractéristiques de l'acte qui font de celui-ci un acte de perception et non pas, par exemple, un acte de remémoration ou d'imagination.

\section{Le noème}

Husserl nomme le noème de l'acte (du grec vón $\mu$ : ce qui est pensé, ce qui est saisi) toutes les caractéristiques d'un acte, aussi bien celles qui déterminent son objet, - s'il en a un -, que celles qui en déterminent l'essence.

Husserl conçoit le noème comme une entité intensionnelle, une « généralisation de la notion de signification au domaine total des actes $\aleph^{I}$. De la même façon que la signification d'une expression linguistique détermine à quel objet celle-ci réfère, le noème détermine ce qu'est l'objet d'un acte - lorsque l'acte a un objet, car certains actes possèdent un noème auquel ne correspond aucun objet.

L'objet de l'acte est fonction du noème, c'est-à-dire que si l'acte a un objet, le noème le détermine de façon univoque. La proposition converse ne peut cependant pas être maintenue car à un seul et même objet peuvent correspondre plusieurs noèmes, et ce dépendamment des différentes manières de faire l'expérience de cet objet (celui-ci pouvant être perçu, imaginé, remémoré, etc.), et dépendamment de son orientation, de notre point de vue, etc.

Pour prendre un exemple de perception, considérons l'acte de voir un arbre. Lorsque nous voyons un arbre, nous ne voyons pas un amalgame de taches colorées, par exemple un certain agencement de brun et de vert; nous voyons plutôt un arbre, un objet matériel ayant une face arrière, des côtés, et ainsi de suite. Certaines de ses parties, par exemple l'arrière, ne peuvent être

I. E. Husserl, Ideen zu einer reinen Phänomenologie und phänomenologischen Philosophie, III, édité par W. Biemel, Husserliana V, La Haye, Martinus Nijhoff, 1952, p. 89 ; tr. fr. par D. Tiffeneau, Idées directrices pour une phénoménologie et une philosophie phénoménologique pures, livre III, Paris, P.U.F., 1993, p.I06. 
vues en même temps que les autres ; mais nous voyons néanmoins une chose ayant une partie arrière. Ce voir est intentionnel, dirigé sur un objet, ce qui veut dire que la partie visible de la chose devant nous n'est considérée que comme la face d'une chose, celle-ci ayant d'autres côtés et d'autres caractéristiques qui sont co-visés de telle sorte que c'est la chose entière qui est considérée, et non pas un simple côté. Le noème est un système complexe de déterminations qui unifie cette multitude d'aspects en un seul objet ayant diverses caractéristiques. Il est important de noter que le noème lui-même est une entité abstraite. Ses différentes composantes correspondent aux différentes caractéristiques de l'objet : à sa couleur, à son inflammabilité, à sa variabilité, etc., mais le noème lui-même ne possède pas ces caractéristiques. Par exemple, comme le remarque Husserl, le noème ne peut brûler ${ }^{2}$.

\section{Deux différences majeures entre le noème de Husserl et l'objet intentionnel de Brentano}

Deux caractéristiques de l'analyse husserlienne de l'intentionnalité sont d'une importance cruciale lorsque nous la comparons à celle de Brentano. Premièrement, la différence la plus évidente réside dans le fait que le noème, auquel est redevable la directionalité de l'acte, n'est pas ce vers quoi l'acte est dirigé. Si le noème était l'objet de l'acte, ou s'il contenait l'objet comme une de ses parties, nous serions alors à nouveau ramenés au dilemme auquel faisait face Brentano : nous n'aurions aucun moyen satisfaisant pour rendre compte d'actes auxquels ne correspond aucun objet, par exemple dans le cas où quelqu'un pense à Pégase.

Deuxièmement, en mettant l'accent sur les structures de la conscience, Husserl accorde plus d'importance que Brentano à la caractérisation de l'intentionnalité de la conscience. Dire, comme Brentano le fit, que l'intentionnalité réside dans cette propriété

2. E. Husserl, Ideen zu einer reinen Phänomenologie und phänomenologischen Philosophie, édité par W. Biemel, Husserliana III, La Haye, Martinus Nijhoff, I950, p. 222 ; tr. fr. par P. Ricoeur, Idées directrices pour une phénoménologie et une philosophie phénoménologique pures, livie I, Paris, Gallimard, 1950, P. 309. 
qu'a la conscience de toujours avoir un objet n'est pas, comme nous l'avons vu, tout à fait exact, ni très éclairant. Ce qu'il faut plutôt dire, c'est que la conscience se comporte toujours comme si elle avait un objet. Cette formulation préserve la valeur de l'intuition de Brentano. Le « comme si » nous amène également à la question posée par Husserl et à laquelle il tenta de répondre par sa phénoménologie: Qu'est-ce, pour la conscience, que d'avoir un objet?

\section{La constitution}

Ces analyses de l'intentionnalité chez Husserl et Brentano suffiront pour le moment. Une fois que l'analyse husserlienne en termes de noème est mise en place, il est aisé d'apercevoir ce que Husserl entend par constitution. Dire que les objets sont constitués par nous signifie simplement qu'ils sont visés de la façon décrite plus haut, à savoir comme ayant normalement un grand nombre d'aspects et de caractéristiques (comme c'est le cas de tous les objets matériels) dont la totalité ne peut jamais être épuisée par l'expérience que nous en faisons. Affirmer que ces objets sont constitués à travers nos actes ne signifie pas qu'ils sont causés par ces actes ou produits par ceux-ci, mais cela indique simplement que dans ces actes, les différentes composantes de la conscience sont reliées de telle sorte que notre expérience se présente comme si elle était celle d'un objet en chair et en os. Tout ce qui détermine l'existence de l'objet correspond ainsi aux composantes de la conscience réunies dans l'acte. Dans le cas des objets physiques, le caractère inépuisable de ce dont nous faisons l'expérience est un trait caractéristique de l'acte et de ce que cela signifie d'être pour un objet physique. Par contre, au sens large du terme « constitution », Husserl dit d'un objet - qu'il soit physique ou non - qu'il

[...] se « constitue » [...] au sein de certains enchaînements immanents à la conscience qui comportent une unité évidente, dans la mesure où ils entraînent par essence la conscience d'un $\mathrm{X}$ identique ${ }^{3}$. 
Incidemment, l'usage que fait ici Husserl, comme à plusieurs autres endroits, de la forme réflexive « un objet se constitue luimême », indique qu'il ne tenait pas l'objet comme étant produit par l'acte. Husserl considérait la phénoménologie comme la première version strictement scientifique de l'idéalisme transcendental, mais il soutenait également que la phénoménologie transcendait la distinction traditionnelle entre le réalisme et l'idéalisme. Ainsi, dans une lettre de 1934 adressée à l'abbé Beaudin, il écrivait : « Aucun "réaliste" conventionnel n'a été aussi réaliste et concret que je le suis, moi, le phénoménologue "idéaliste" (mot dont je ne fais d'ailleurs plus usage) $»^{4}$. Husserl n'a pas cherché à « réduire » la réalité à la conscience. Selon lui, dans notre expérience du monde, il y a un certain donné qui intervient, un élément étranger à l'ego : la hylè. Cependant, cet article ne nous permettera pas d'entrer plus avant dans cet aspect de sa philosophie.

\section{La philosophie de Heidegger en tant que traduction de celle de Husserl}

Suite à cette brève esquisse de la position de Husserl sur la constitution, tournons-nous maintenant vers Heidegger. Nous reviendrons, à la fin de cet article, à la position que défend Husserl dans certains de ses écrits inédits.

La philosophie de Heidegger semble à première vue fort différente de celle de Husserl. Les thèmes traités, le vocabulaire ainsi que le style d'écriture sont différents. Heidegger reconnaît toutefois avoir été fortement influencé par Husserl. Il définit t̂tre et Temps ${ }^{5}$, texte sur lequel je me concentrerai, comme un travail phénoménologique, et il déclare explicitement y utiliser la méthode phénoménologique.

La clef de ce problème, qui ouvre également, selon moi, à la compréhension de l'articulation de la philosophie heideggerienne, réside dans le fait que celle-ci est fondamentalement isomorphe à celle de Husserl. Lorsque Husserl parle de l'ego, Heidegger parle du Dasein, et lorsqu'il parle du noème, Heidegger

4. Lettre citée par Iso Kern dans son livre Husserl und Kant, La Haye, Martinus Nijhoff, I964, p. 276.

5. M. Heidegger, Être et Temps, tr. fr. par E. Martineau, Paris, Authentica, 1985. 
parle de la structure de l'être-au-monde du Dasein, et ainsi de suite. Husserl remarqua également cela à plusieurs endroits en marge de son exemplaire d'Être et Temps ; il nota que Heidegger traduisait tout simplement sa phénoménologie dans une autre terminologie. Ainsi, par exemple, Husserl écrit en marge de son exemplaire à la page 13 : « Heidegger transpose ou transforme la clarification phénoménologique constitutive de tous les domaines d'entités et d'universels, l'ensemble de la région monde, en une anthropologie. Toute la problématique est une transposition ; à l'ego correspond le Dasein, etc. Conséquemment, tout plonge dans une profonde confusion et perd ainsi sa valeur philosophique ». De la même façon, Husserl remarque à la page 62 de son exemplaire : « Ce qui est dit ici est ma propre théorie, mais sans justification plus profonde $\gg$.

En affirmant que la philosophie de Heidegger est essentiellement isomorphe à celle de Husserl, je ne prétends pas qu'il y a isomorphisme en tout point. Comme nous le verrons, il y a au moins deux points importants sur lesquels leur position diffère. Mais cet isomorphisme va au-delà des seuls éléments de base que Husserl et Heidegger ont en commun avec Kant, par exemple. Les notions fondamentales de leur philosophie sont interreliées jusque dans les moindres détails, détails qui ne figurent même pas chez Kant. Nous ne pourrons nous livrer, ici, à une justification de cette affirmation par une analyse détaillée des textes. Nous nous référerons seulement aux principaux traits de cet isomorphisme dans le but de réaliser nos deux intentions fondamentales : faciliter la présentation de certaines idées de $\mathrm{Hei}^{-}$ degger sur la base de ce que nous venons de voir à propos de Husserl et mieux faire voir, en les circonscrivant, les différences majeures entre les deux.

\section{f. Une esquisse de la philosophie de Heidegger}

Si on interprète les idées essentielles de la philosophie de Heidegger comme une traduction de la pensée de Husserl, elle se resume alors à une tentative d'elucider ce que c'est que d'être. Être, pour une chose dans le monde, consiste à être constituée. Afin de comprendre ce que c'est que d'être constitué, nous devons nous concentrer sur ce qui constitue, à savoir l'ego ou le Dasein. Heidegger utilise le terme « Dasein» de façon à accentuer le 
double rôle de l'ego : il est là $[d a]$, chose parmi les choses dans le monde, mais il est également source d'Être [Sein], ce qui constitue les choses dans le monde. Tandis que être, pour les choses, consiste à être constitué, le Dasein est autant constitué que constituant, et c'est dans ce dernier trait que réside sa marque distinctive. La réponse la plus appropriée à la question « Qu'estce que le Dasein ? » est : « il constitue ».

Il y a ainsi deux modes d'être : être constitué et constituer. Pour caractériser le second de ces modes, Heidegger utilise le terme « existence ». L'« essence » du Dasein, c'est-à-dire la réponse appropriée à la question « Qu'est-ce que le Dasein? ?», est ainsi l'existence. Ces deux aspects du Dasein chez Heidegger, à savoir qu'il est dans le monde et qu'il le constitue, correspondent respectivement à l'ego empirique et à l'ego transcendantal chez Husserl. Dans une lettre à Husserl ${ }^{6}$, Heidegger reprocha à ce dernier de dédoubler l'ego et de développer, de cette façon, une théorie schizophrénique de l'ego. Husserl répliqua que l'ego empirique et l'ego transcendantal ne constituent pas deux ego, mais plutôt deux aspects du même ego. Comme Husserl l'a écrit, il n'y a qu'un ego : il est dans le monde et il le constitue. Il semble donc y avoir sur ce point un parallélisme beaucoup plus complet entre Husserl et Heidegger que ce dernier ne semblait le croire.

\section{La position de Heidegger sur la constitution}

Mais que signifie maintenant constituer? Nous avons déjà vu ce que Husserl entendait par «constitution ». La théorie de Heidegger est plus complexe. Par «constitution », il désigne l'être-aumonde en tant que mode spécifique au Dasein d'être dans le monde. Il ne s'agit pas d'être dans le monde tel que l'eau est dans un verre ou une chose parmi les choses, mais plutôt de se rapporter au monde de toutes les manières possibles qui sont propres au Dasein. Être et Temps vise en bonne partie à dégager ces manières par lesquelles le Dasein peut se rapporter au monde.

L'une de celles-ci, celle considérée par Husserl, consiste à le faire d'une façon théorique. C'est le cas dans les sciences, lorsque

6. Il s'agit de la lettre du 22 octobre i927, tr. fr. par J.F. Courtine dans Heidegger, Paris, Cahier de l'Herne, Livre de poche, 1983. p. 66-69. 
nous étudions les différents objets et leurs propriétés, les percevons et élaborons des théories à leur propos. Heidegger écrit qu'en entretenant un tel commerce avec les objets, nous faisons l'expérience de ceux-ci comme étant « là-devant » [Vorhandenheitl. Depuis Aristote, cette façon de se rapporter au monde s'est avérée la préférée des philosophes pour concevoir notre relation au monde; en fait, elle a été si favorisée que la plupart des philosophes n'ont pas songé à en considérer d'autres, et s'ils l'ont fait, ils les ont toujours tenues pour secondaires et fondées sur l'attitude théorique. On a souvent prétendu que l'activité pratique présuppose une compréhension théorique du monde, que l'action présuppose que l'on ait une idée de ce qui arrivera si on fait ceci ou cela. Heidegger rejette cela. Il considère que les différentes manières pratiques d'être en rapport avec le monde sont plus fondamentales que les modes théoriques. Comme il l'écrit, nous faisons originairement l'expérience des choses dans le monde en tant qu'« à-portée-de-la-main » [Zuhandenheitl, en tant qu'outils ou outillage dont nous faisons usage. Ils sont ce qu'ils sont en fonction de leur position au sein du contexte global de l'activité humaine et de son schème de moyens et de fins. Être un marteau, par exemple, consiste à être un outil servant à enfoncer des clous, à écraser des choses, etc. Ce n'est que lorsque nous pratiquons la réflexion théorique que le marteau peut être conçu comme un objet d'une certaine longueur, d'une certaine forme, de telle couleur, de tel poids, etc. L'attitude théorique est « parasitaire » de l'activité pratique : c'est à travers notre commerce pratique avec le monde que les objets sont isolés de ce qui les entoure pour s'individualiser en objets qu'il nous est ensuite possible de sonder théoriquement.

Je n'entrerai pas dans le détail de l'analyse heideggerienne des multiples manières par lesquelles nous pouvons nous rapporter au monde. Elle anticipe, sous plusieurs aspects, quelques-unes des dernières analyses de Wittgenstein sur les « formes de vie ». En fait, la majeure partie de ce que Wittgenstein a écrit sur la signification des mots, en tant que produit de leur usage, est parallèle aux observations de Heidegger concernant la «signification »des objets, à savoir ce que c'est que d'être pour divers objets. Voilà à quoi il faut s'attendre si, comme le prétendait Husserl, les questions de constitution et de signification sont intimement 
liées - le noème étant, comme nous l'avons noté, « une généralisation de la notion de signification à l'ensemble du domaine des actes $\gg$.

\section{Anticipations des idées heideggeriennes par Husserl}

Comme je l'ai mentionné en introduction, je considère l'idée de Heidegger voulant que toute l'activité humaine joue un rôle dans notre constitution du monde, ainsi que son analyse de ce phénomène, comme ses principales contributions à la philosophie. C'est aussi principalement sur ce point que la pensée de Heidegger dépasse celle de Husserl ${ }^{7}$. Interpréter, comme je l'ai fait, Heidegger à partir de Husserl permet d'indiquer plus aisément en quoi consiste la contribution de Heidegger. Comme nous l'avons vu, dans ses écrits publiés, Husserl concentre presque toute son attention sur le mode théorique par lequel nous nous référons au monde. La plupart de ses exemples portent sur la perception. Selon Husserl, cette approche est nécessaire car la perception est un type d'acte relativement simple et primaire qui figure comme composante d'actes plus complexes, comme par exemple la volition et l'évaluation. Cependant, après son arrivée à Freiburg en 19i6, plus précisément vers la fin des années dix et au début des années vingt, il est clair que Husserl devint alors de plus en plus conscient du rôle important joué par l'activité pratique dans notre rapport au monde. Ainsi, dans les notes destinées à une nouvelle édition des Idées directrices, il remarque que l'attitude pratique devrait également intervenir à divers endroits où, dans la première édition, il n'est question que de l'attitude théorique. Dans les trois leçons sur Fichte ${ }^{8}$ professées en novembre Igr7, et qu'il

7. Un autre point majeur sur lequel il s'écarte de Husserl réside dans son rejet de la " réduction transcendantale », à savoir la réflexion particulière par laquelle, selon Husserl, nous étudions la structure de notre propre conscience, le noème ou la constitution. Heidegger prétendait qu'une telle réduction est impossible et que nous ne pouvons étudier cette structure que « de l'intérieur », c'est-à-dire en devenant conscient que nous sommes constamment au milieu de celle-ci. Cette conscience se manifeste normalement lors du bris d'un outil familier, lors d'un face à face avec la mort, etc. Cependant, nous n'examinerons pas ici les positions de Heidegger sur la réduction transcendantale, ni la méthode alternative qu'il emploie pour son analyse phénoménologique.

8. E. Husserl, " Fichtes Menchheitsideal 》, dans Aufsätze und Vorträge (IgII-192I), 
répéta deux fois en $19 \mathrm{I} 8$, il souligne que pour ce philosophe, être un sujet consiste à être celui qui agit et de la sorte, I... être objet pour un sujet consiste à être un produit de l'action. Si nous remontons jusqu'à une certaine origine, rien ne précède l'action. Lorsque nous considérons ce qu'il est permis de nommer l'histoire $d u$ sujet, l'origine n'est pas une question de fait [Tatsachel, mais une action ITathandlungl, et c'est ainsi seulement que nous pouvons penser une histoire. Être un sujet consiste eo ipso à avoir une histoire, un développement. Être un sujet consiste non seulement à agir, mais aussi nécessairement à poursuivre d'action en action : du produit d'une action, par une nouvelle action, à un nouveau produit ${ }^{9}$.

Il s'agit là, pour Husserl, d'« une suite infinie de buts, d'objectifs et de tâches » auxquels se rapportent nos actions ainsi que leurs produits. Cette idée, présentée par Husserl en rgi7 dans cette terminologie, ressemble fort à l'idée d'un réseau de renvois IVerweisungenl ou de relations d'« en-vue-de » au sein duquel s'insèrent nos activités et nos outils, idée présentée dix ans plus tard par Heidegger dans $\hat{E} t r e$ et Temps (particulièrement dans les sections 17 et 18 ). Incidemment, il y a un autre parallèle terminologique frappant entre Heidegger et Husserl, et ce dans le terme clef «Sorge » (souci) qui pour Heidegger, est commun à toutes les manières par lesquelles le Dasein se rapporte au monde. Ce même terme figure dans un manuscrit de Husserl daté de ig25 et dans lequel l'activité humaine se divise en deux types principaux : Spiel (jeu) qui est sans but, et la « praxis sérieuse au sein du Sorge (souci) sérieux et originel $\gg$.

Nous devrions aussi noter que Fichte, sur qui portaient les leçons de Husserl de 1917, semble avoir été le premier à mettre la notion d'action en rapport avec celle de constitution; il a peutêtre ainsi été, sur ce point, une source d'inspiration commune à Husserl et à Heidegger.

T. Nenon et H.S. Sepp (dir.), Husserliana XXV, Dordrecht, Martinus Nijhoff, I987, p. 267-293-

9. Ibid., p. 275, (F I 22, 9a-20).

Io. Manuscrit (B I 21, IV, 52) 


\section{Comment les actions constituent le monde pour Husserl}

Le manuscrit « Science et Vie » (I9I7-19I8), qui date à peu près de la même époque que les leçons sur Fichte, contient l'expression la plus explicite de la pensée de Husserl sur la manière par laquelle le monde se constitue à travers notre activité pratique. Il s'agit d'un manuscrit très important, premièrement parce qu'il est le plus instructif jamais écrit par Husserl sur cette question, et deuxièmement parce qu'il remonte à I9I7-ı8, c'est-à-dire bien avant que Heidegger n'entreprenne d'écrire Être et Temps (publié en 1927, soit dix ans après les leçons de Husserl sur Fichte). Dans une section de ce manuscrit portant sur l'« Analogie », Husserl commence par expliquer brièvement comment notre conception théorique d'un objet se fonde, par analogie, sur des expériences antérieures. Ces analogies, ou similarités, servent de guides [Leitfadenl à nos anticipations : « une prescription analogique » lanalogische Vorzeichnungl a lieu. Suite à son explication de la constitution dans le domaine théorique, Husserl poursuit en introduisant la notion d'aperception pratique.

Ceci conduit ensuite au domaine pratique et à l'aperception pratique : on se rapporte à ce qui est aperçu par analogie de la même façon que l'on se rapporte à ce qui lui est analogue. On le « manipule » [behandelt] d'une façon similaire, on essaie d'établir des fins similaires lui correspondant ou de l'utiliser d'une façon similaire comme un moyen, on l'oriente d'une façon analogue, etc. L'extrapolation analogique se confirme à travers l'activité pratique, conduisant ainsi à des aperceptions théoriques et pratiques plus fermes que les précédentes ${ }^{\text {II }}$.

Nous pouvons conclure de ce passage que pour Husserl, la constitution se déploie d'une façon parallèle dans les champs pratique et théorique, et ce sur la base de similarités et d'analogies. Ces analogies donnent lieu à des extrapolations qui constituent le noème et, de ce fait, constituent aussi l'objet. La différence entre le domaine pratique et le domaine théorique tient au fait que dans ce dernier, les extrapolations concernent les propriétés factuelles de l'objet, alors que dans le domaine pratique, les extrapolations concernent plutôt l'utilité de l'objet au sein de notre réseau de moyens et de fins. Cette explication de la manière dont 
l'action constitue le monde, soit par une extrapolation fondée sur des analogies établies dans nos rapports pratiques avec les choses, me semble être la plus éclairante donnée sur ce point par Husserl ou Heidegger.

Rien n'indique cependant que Husserl aurait conçu l'aperception pratique comme étant fondée sur une perception de similarités factuelles entre les choses, et dont on inférerait ou anticiperait qu'elles pourraient également être traitées de manière semblable pour des fins pratiques. Il ne prétend pas non plus que nous percevons d'abord la praxis pour ensuite anticiper, par un procédé cognitif, des praxis similaires. Husserl n'importe pas l'ordre du voir et du savoir dans un domaine où l'agir et le savoir-faire sont fondamentaux. Pour Husserl, l'aperception pratique réside dans notre capacité d'extrapoler des caractéristiques pratiques de la même façon qu'il nous est possible d'extrapoler des caractéristiques théoriques : lorsque deux choses se sont révélées similaires dans certaines applications pratiques, nous en venons à les considérer comme étant également similaires dans plusieurs autres applications pratiques. Selon Husserl, la perception et la vision n'ont aucune primauté sur l'action. Rappelons-nous du passage cité plus haut où il écrit : «l'origine n'est pas une question de fait, mais une action $\gg$.

\section{Parallèles entre l'activité théorique et l'activité pratique}

\section{Elles sont toutes deux régies par des intérêts}

Ce parallélisme entre la constitution théorique et la constitution pratique est compatible avec l'observation que fait Husserl dans plusieurs autres manuscrits et suivant laquelle l'activité théorique n'est qu'un type d'activité humaine parmi plusieurs autres. Comme toutes les autres activités, elle vise, par exemple, à servir nos fins et nos desseins. Toute activité, y compris l'activité théorique, est sous la gouverne de nos intérêts ; nous avons un intérêt particulier, purement théorique ou purement doxique ${ }^{\mathrm{I2}}$, pour la connaissance et pour la formation de concepts et de jugements portant sur ceux-ci ${ }^{13}$. « L'intérêt théorique inhibe tout 
autre intérêt d'ordre personnel [...] le "spectateur désintéressé" met ainsi hors jeu tout autre intérêt au sens courant $\gg^{\mathrm{I}}$. Husserl écrit également que « l'attitude de la pure observation, l'attitude "théorique", est [...] une attitude pratique particulière $[. ..] \gg^{\mathrm{I}} 5$. Husserl souligne que

[... l'intérêt principal de la connaissance peut être subordonné à un intérêt pratique, mais il peut aussi s'agir d'un intérêt théorique pur, un intérêt pour la chose même, « telle qu'elle est réellement $»^{16}$.

\section{Anticipations et remplissement}

Revenons une fois de plus sur le parallélisme entre les anticipations théoriques et pratiques. Dans son manuscrit « Introduction à la philosophie $»^{17}$, Husserl mentionne l'« anticipation pratique », et dans plusieurs autres manuscrits, il discute de l'horizon pratique des possibilités liées à nos actions ${ }^{\text {I8 }}$. Même chose dans La crise des sciences européennes et la phénoménologie transcendantale oị l'activité théorique est considérée comme un type de praxis. Il y souligne également que le « monde de la vie » constitue l'horizon au sein duquel prend place toute activité humaine, qu'elle soit ou non théorique ${ }^{\mathrm{Ig}}$.

Dans un autre manuscrit datant de 1922-23, Vormeditationen über die Idee der Philosophie, Husserl précise que l'on peut parler d'un remplissement de l'intention non seulement dans le domaine théorique, mais également dans le domaine pratique. Lorsqu'il s'agit du domaine théorique, notre intention se remplit, au moyen de la perception par exemple, remplissement que nous nommons dans ce cas « évidence ». Dans d'autres domaines, comme ceux des actions et des sentiments, le remplissement est différent, et il dépend dans chaque cas du type d'intention impliqué et de ce qui pourra satisfaire celle-ci. Suivant en cela Fichte, Husserl emploie « félicité » [Seligkeit] comme terme commun à

I4. Manuscrit (B I 2i.IV.I4 encore, de ig25).

15. Manuscrit (F I 44 (I.Teil) de 1926-27).

I6. Manuscrit (A VI 26, I8a, de 1928)

17. Manuscrit (F I 29.186.bi.94Rs.).

18. Voir par exemple (A V Io (I.Teil), 2, de 193i).

19. Edmund Husserl, Die Krisis der europaischen Wissenschaften und die transcendentale Phänomenologie, édité par W. Biemel, Husserliana VI, La Haye, Martinus Nijhoff, 1976. p. 145 ; tr. fr. par G. Granel, La crise des sciences européennes et la phénoménologie transcendantale, Paris, Gallimard, 1976, p. 16r. 
tout type de remplissement de l'intention d'un acte ${ }^{20}$. On retrouve ici, comme à d'autres endroits chez Husserl, la tendance à fusionner deux notions d'intention que Brentano séparait clairement : l'intention au sens de la directionalité d'un acte vers un objet, et l'intention au sens pratique d'un but ou objectif d'une action. Cette fusion est particulièrement apparente dans un manuscrit de 1928 où Husserl écrit :

De la même façon que se retrouve un objectif « pratique » dans le cas d'une décision volontaire et d'une action par laquelle quelque chose est fait l...l il y a ainsi généralement un but dans tous les cas d'intentionnalité dirigée, donc également dans le cas d'une représentation et d'une pensée ${ }^{2 I}$.

Cette fusion de deux notions, très différentes selon Brentano, peut aisément être considérée comme une confusion. Cependant, Husserl prend bien soin de souligner qu'il y a d'importantes similarités entre ces deux notions:

[...] tant que l'action n'a pas mené au but vers lequel elle est dirigée, nous sommes toujours conscients de ce but en tant que ce vers quoi tend l'action ; mais le but est transcendant - une réalité transcendante qui n'a pas encore été réalisée ${ }^{22}$.

\section{Conclusions}

Je terminerai cet article par une remarque historique et une remarque systématique.

\section{Husserl et Heidegger}

La remarque historique concerne la relation entre Husserl et Heidegger. Il me semble y avoir toutes les raisons de soutenir, comme le fit Husserl, que le cadre principal de la philosophie développée par Heidegger dans Être et Temps constitue une traduction de sa propre philosophie. En considérant ainsi ce texte comme une traduction, il devient plus facile de déterminer sur quels points ces deux philosophes s'entendaient, et sur quels autres ils divergeaient. Le point de divergence principal me semble résider dans l'argument de Heidegger suivant lequel le monde est constitué par toutes sortes d'activités humaines, alors que

\footnotetext{
20. Manuscrit (B I 37.31a).

21. Manuscrit (F I 44 [2.Teil], 123-b).

22. Ibid.
} 
Husserl soutenait, dans ses écrits publiés, que le monde se constituait principalement à travers notre activité théorique. Il devrait cependant ressortir clairement des passages cités des manuscrits inédits de Husserl, que ce dernier avait des idées similaires à celles de Heidegger, et ce bien avant la publication de Être et Temps. Ces idées prirent racine chez Husserl, peu après son arrivée à Freiburg où il rencontra Heidegger en igi6. Il est possible que Husserl ait attiré Heidegger dans cette direction « pratique ». Quoi qu'il en soit, ces idées commencèrent à se retrouver dans les manuscrits de Husserl bien avant que Heidegger n'entreprenne l'écriture de Être et Temps. Cependant, il est également possible que Husserl ait été attiré dans cette direction suite à des discussions avec le jeune Heidegger. Ni l'un ni l'autre ne parle d'une telle influence sur ce point, et il ne semble y avoir aucune autre information disponible concernant la direction dans laquelle s'accomplit cette influence. Vraisemblablement, elle alla dans les deux sens.

Ce qui est important de retenir, c'est que l'idée de la constitution du monde par l'activité pratique fut pour Heidegger un thème principal autour duquel s'organisa toute sa pensée, alors que pour Husserl, cette idée ne semble s'être imposée qu'après coup et d'une manière plutôt fragmentaire sans qu'elle fût insérée dans le corps même de ses écrits.

\section{Le corps est-il nécessaire à l'explication de la constitution ?}

Ma seconde remarque est systématique et en rapport étroit avec ma remarque historique : tout au long de ses écrits, y compris ses manuscrits, Husserl insiste sur l'idée que c'est par notre conscience que nous constituons le monde. Husserl tenait toutes nos actions et activités pour des « activités de la conscience » [Bewußtseinstätigkeiten]. Je cite ici un de ses manuscrits de 1922-23 (Vormeditationen über die Idee der Philosophie) :

Nous ne considérons plus maintenant l'homme comme la simple unité inductive et externe d'un corps et d'un esprit, en tant qu'objet réel, en soi dichotomique, s'inscrivant dans un contexte causal spatio-temporel ; nous le considérons maintenant de la façon par laquelle nous nous considérons lorsque nous disons Je, ou lorsque dans une relation Je-Tu, nous tenons l'autre pour un Tu et que nous lui demandons quelque chose, etc. Nous considérons donc l'homme comme un Je-sujet qui, en tant que tel « se rapporte à son monde ambiant » en tant que Je - c'est-à-dire qu'il perçoit, fait des expériences, se remémore, pense, sent, veut et agit en tant que Je qui, dans 
toutes ces « activités de la conscience », a conscience d'un monde ambiant et est conscient d'être affecté par celui-ci. Face à ce monde duquel il est conscient et qui l'affecte, il prend consciemment position d'une manière théorique aussi bien que pratique ${ }^{23}$.

Nous suffit-il de ne considérer que la conscience lorsque nous cherchons à clarifier comment nos actions contribuent à constituer le monde? Nos actions n'impliquent-elles pas aussi notre corps, et ce de telle sorte qu'il devienne nécessaire d'en tenir compte dans toute discussion concernant la constitution? En différents endroits, aussi bien de ses écrits publiés qu'inédits, Husserl explicite le rôle joué par le corps dans la constitution. Il est le « centre de toutes les orientations dans le monde » [Zentrum aller Orientierungen $\left.\right|^{24}$, le «centre de mon activité corporelle $»^{25}$ et « l'objet direct de toute expérience pratique et de toute praxis qui agit et produit [handelnden-Wirkenden Praxisl » (ibid.). Husserl souligne également à maintes reprises le rôle que jouent les kinesthèses au sein de la perception. Ainsi, par exemple, dans le manuscrit «Introduction à la Philosophie » datant de 1922-23, il écrit :

I...J le fait d'avoir un corps [Leiblichkeitl joue un rôle essentiel dans toute perception, et ce en partie à cause des kinesthèses qui sont constamment produites, et en partie aussi à cause de ce que je nomme la perfection de mes sens, mon sens de la vue, du toucher, etc. $^{26}$.

On retrouve également plusieurs passages semblables dans sa Krisis.

Mais qu'en est-il des mouvements routiniers de mon corps dont je ne suis pas normalement conscient, comme par exemple le mouvement de mes jambes lorsque je marche, etc. ? Ces mouvements ne contribuent-ils pas aussi à la manière par laquelle je constitue le monde? Ont-ils un corrélat au sein de la conscience? De tels mouvements routiniers ne devraient probablement pas être considérés comme des actions, mais si tel était le cas, cela ne montrerait-il pas simplement que de tels mouvements, et non pas seulement les actions, jouent un rôle dans notre constitution du monde?

\footnotetext{
23. Manusctit (B.I 37.15).

24. Manuscrit (B I 37, I26).

25. Manuscrit (D 13 VII, I).

26. Manuscrit (F 1 29, 22I).
} 
Husserl insiste sur le fait que toute constitution n'est que par la conscience. En questionnant constamment la genèse de la constitution, c'est-à-dire comment nous pouvons avoir les noèmes que nous avons, il peut avoir souligné l'importance du corps dans le processus nous menant à constituer le monde tel que nous le faisons, mais il a toujours maintenu que le produit de la constitution, le monde tel que je le conçois à quelque moment donné, doit être le corrélat de la structure de ma conscience à ce même moment. On doit donc tenir compte, comme Husserl le fait, du corps afin d'expliquer la genèse de la structure de la conscience. Toutefois, ceci n'infirme aucunement, selon lui, que le monde soit constitué par cette conscience.

Heidegger, ainsi que Merleau-Ponty, semble soutenir qu'il faille tenir compte du corps afin non seulement d'expliquer la genèse de la constitution, mais également pour rendre compte de ce qu'est la constitution comme telle. Pour eux, le monde n'est pas seulement constitué par la conscience, mais par un ego à la fois corporel et conscient, ou, dans la terminologie heideggerienne, par le Dasein. Pour Husserl aussi, l'ego est à la fois corporel et conscient ; mais selon lui, seule la conscience est nécessaire pour expliquer ce en quoi consiste la constitution.

\section{Université d'Oslo et Université de Stanford}

(Traduction francaise par P. Saudrais et J.-F. Campeau) 